\title{
A Noncontact Method for the Detection and Diagnosis of Surface Damage in Immersed Structures
}

\author{
Y. Sidibe, ${ }^{1}$ F. Druaux, ${ }^{1}$ D. Lefebvre, ${ }^{1}$ F. Leon, ${ }^{2}$ and G. Maze $^{2}$ \\ ${ }^{1}$ GREAH, Normandie Université, 75 rue Bellot, 76058 Le Havre, France \\ ${ }^{2}$ LOMC, UMR CNRS 6294, Normandie Université, 75 rue Bellot, 76058 Le Havre, France \\ Correspondence should be addressed to Y. Sidibe; yasidibe@gmail.com
}

Received 14 October 2014; Accepted 16 April 2015

Academic Editor: Emil Manoach

Copyright ( $) 2015$ Y. Sidibe et al. This is an open access article distributed under the Creative Commons Attribution License, which permits unrestricted use, distribution, and reproduction in any medium, provided the original work is properly cited.

\begin{abstract}
Detection and diagnosis method is proposed for surface damage in immersed structures. It is based on noncontact ultrasonic echography measurements, signal processing tools, and artificial intelligence methods. Significant features are extracted from the measured signals and a classification method is developed to detect the echoes resulting from surface damage in an immersed structure. The identification of the damage is also provided. Gaussian neural networks trained with a specific learning algorithm are developed for this purpose. The performance of the method is validated by laboratory experiments which indicate that this method could be suitable for the monitoring of inaccessible systems like marine turbines whose unavailability causes severe economic losses.
\end{abstract}

\section{Introduction}

The structural damage detection and diagnosis methods are helpful in systems monitoring. They combine various disciplines as material science, signal processing, wave propagation, automatics, and computer science. A state of art can be found in Mechbal et al. [1]. In particular, vibration active control [2] and modal control strategies [3] have been used for mechanical structures monitoring. In this paper, surface damage in immersed structures is considered, especially in metal plates. Our objective is to perform damage detection and classification for such plates. The motivation for this work is to propose tools for the early remote diagnosis of mechanical damage in the surface of immersed offshore marine turbines or other metallic structures. Due to the environmental hostility, the blades of the marine turbines are subject to such surface damage [4]. The inspection and maintenance of such equipment is a complicated and expensive task and the remote monitoring is a promising alternative to the usual in situ inspection by humans or robots. The proposed approach could be adapted so that it could be used in a ship that navigates in the neighborhood of the inspected sites. For that purpose, ultrasonic pulses are generated through a transducer that has no contact with the structure under investigation. Pulses propagate through the water and excite the structure where Lamb waves are generated. These waves, discovered in 1917 by Horace Lamb, have been the subject of multiple studies in Nondestructive Testing (NDT) area [5-7]. Lamb waves are guided waves that propagate in thin plates or curved shells. They propagate over the structure and reflect at the edges. Surface damage also changes the wave propagation. As a consequence, the Lamb waves are sensitive to such damage. The measurement of the backscattered signal contains information to characterize the damage in the structure. Consequently, the proposed method should assist in scheduling the maintenance operations for immersed structures.

The method involves the following steps. At first, specific echoes from the structures edges are detected and a useful range limited by these echoes is extracted. The most significant features are then extracted and clustered for damage detection and diagnosis purposes. Features extraction is obtained with usual statistical analysis, principal components analysis, and signal segmentation methods. The classification of the patterns is performed using Gaussian neural networks. The proposed approach has several advantages. In comparison with numerous other NDT methods that require several sensors [8] or a sophisticated data acquisition system [4], 


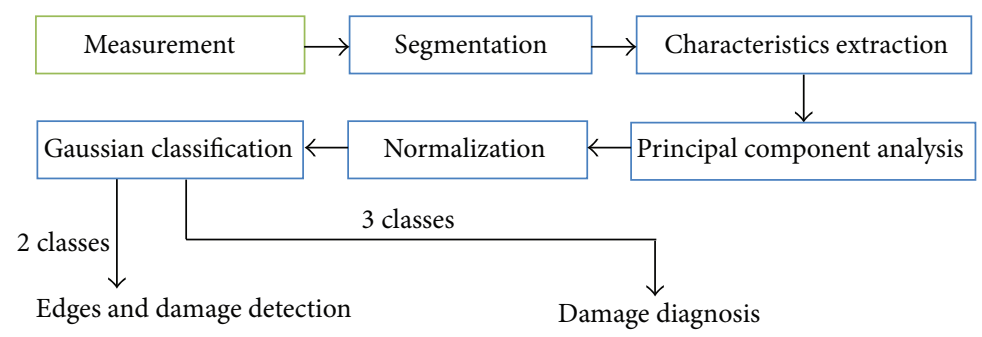

FIGURE 1: (Colour online) Detection and diagnosis scheme for surface damage in immersed structures.

the present contribution is based on measurements issued from a single sensor. The advantages of Gaussian classifiers have been detailed in $[9,10]$. The whole process is detailed in Figure 1.

The paper organization is as follows. In Section 2, the preprocessing tools and the classification network are presented. Section 3 details the detection and diagnosis method. The experimental measurements and results are discussed in Section 4. Conclusions and perspectives are drawn in the last section.

\section{Classification of Acoustic Signals}

The main objective of this section is to present some tools used to separate samples into two or more classes. For the proposed application, the samples are acoustic measurements of metallic plates with the same dimensions and the same physical characteristics. The aim of the proposed classification method is to detect echoes issued from the plate edges and from surface damage.

2.1. Signal Preprocessing. Acoustic signal measurements are obtained as temporal series of data collected under specific geometric specifications such as the distance and the orientation of the structures of interest with respect to the position of the transducer that generates the pulses and receives the measurements.

The 1st stage separates each signal measurement $S$ into $N$ segments $I_{k}, k=1, \ldots, N$. Each segment is of size $w$ (number of points). This stage is useful to enhance the information about the edges of the plate and the considered damage.

The 2nd stage extracts significant features from each segment with the computation of specific parameters in time domain. A total number of 8 features are computed from each segment $I_{k}, k=1, \ldots, N$. These features are the mean value $\left(m_{k}\right)$, the standard deviation $\left(\sigma_{k}\right)$ that represents the dispersion, the kurtosis $\left(\operatorname{kur}_{k}\right)$ that shows how the distribution is sharp or flat relatively to a normal distribution, the skewness $\left(\mathrm{sk}_{k}\right)$ that measures the asymmetry around the mean, the maximal magnitude $\left(\mathrm{mm}_{k}\right)$, the energy $\left(E_{k},\right)$ of the segment, the sum of quadratic variations $\left(\operatorname{sqvr}_{k}\right)$, and the number of sign switches $\left(\mathrm{ns}_{k}\right)$. For each segment $I_{k}$, the extracted features are summed up in vector $P\left(I_{k}\right)=\left(x_{n}\right)$, $n=1, \ldots, 8$.

The 3rd stage combines the extracted features in order to select the most significant ones. For this purpose, the Principal Component Analysis (PCA) is used [11, 12]. The correlation matrix $M^{T} \cdot M$ is computed. Each line of matrix $M$ represents a given segment $I_{k}$ and each column of matrix $M$ represents a given feature. The features are centered on their mean value and reduced according to their variance. The eigenvalues $\lambda_{n}$ and eigenvectors $u_{n}, n=1, \ldots, 8$, of $M^{T} \cdot M$ are performed and the projection over the most significant principal components is obtained with matrix $U$ of selected eigenvectors [13]. In this paper, the two most significant principal components (i.e., the eigenvectors associated with the eigenvalues of maximal magnitude) are selected. For each parameter vector $P\left(I_{k}\right)$, the projection $P^{\prime}\left(I_{k}\right)=U \cdot P\left(I_{k}\right)$ is computed.

The 4th stage transforms $P^{\prime}\left(I_{k}\right)$ into $x=P^{\prime \prime}\left(I_{k}\right)$ with $x=$ $\left(x_{n}^{\prime \prime}\right), n=1,2$, according to normalization between 0 and 1 :

$$
x_{n}^{\prime \prime}=\frac{x^{\prime \prime}-x_{\text {Min }}^{\prime \prime}}{x_{\text {Max }}^{\prime \prime}-x_{\text {Min }}^{\prime \prime}}, \quad n=1,2,
$$

where $x_{\text {Min }}^{\prime \prime}$ and $x_{\text {Max }}^{\prime \prime}$ are, respectively, the minimal and maximal values of component $x^{\prime \prime}$. Finally, the vectors $P^{\prime \prime}\left(I_{k}\right)$ are separated into a learning set $(\mathbf{L})$ that will be used to determine the parameters of all preprocessing stages and to train the classification network (detailed in next section) and a validation set (V) that will be used to evaluate the performance of the proposed method. Both sets contain the same number of measurements.

2.2. Gaussian Neural Network Classifier. The classification of the parameter vectors $P^{\prime \prime}\left(I_{k}\right)$ is obtained with a Gaussian neural network that has a feed forward architecture with one input layer with 2 linear nodes, one hidden layer composed of $N_{G}$ Gaussian nodes, and one output layer with $N_{C}$ output linear nodes. It performs a classification into $N_{C}$ classes. The basic idea of the Gaussian neural network classification is to associate each Gaussian function with a single class and evaluate the class of each data by computing the $N_{G}$ Gaussian nodes activations. The activation of the Gaussian functions is defined by

$$
G_{j}\left(P^{\prime \prime}\left(I_{k}\right)\right)=\frac{1}{d_{j} \sqrt{2 \pi}} e^{-\left\|P^{\prime \prime}-v_{j}\right\|^{2} / 2 d_{j}^{2}}, \quad j=1, \ldots, N_{G},
$$

where $d_{j}$ and $v_{j}$ are, respectively, the dispersion and the center of the $j$ th Gaussian function. The use of Gaussian functions as classification nodes is motivated because the separation of 


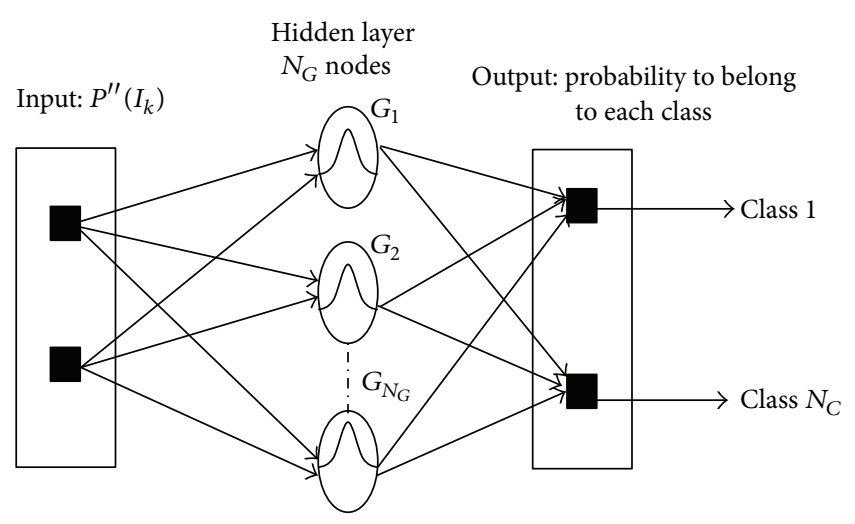

Figure 2: (Colour online) Gaussian neural networks.

the Gaussian surfaces are either circle arcs or straight lines depending on the value of the Gaussian function parameters [14]. The same kind of Gaussian neural networks will be used either to detect the echoes of the structure edges in order to extract the useful range (with $N_{C}=2$ ) or to detect and identify the surface damage (with $N_{C}$ that is equal to the number of types of damage). The architecture of the Gaussian neural network is described in Figure 2.

2.3. Learning Algorithm. A supervised learning algorithm is proposed to train the Gaussian neural network. This algorithm computes iteratively the position of centers and dispersion of the Gaussian functions. It also increases the number of Gaussian nodes when required. Then, a stopping criterion is performed with respect to the maximal number of iterations $K_{\max }$. At each iteration, the misclassification rate is computed. For this purpose, $E(j, q)$ is defined as the proportion of learning data of type $j$ that activate Gaussian nodes associated with the class $q$. The mean error of classification is given by

$$
E_{m}=\frac{1}{N_{C}\left(N_{C}-1\right)}\left(\sum_{j=1, \ldots, N_{C}, q=1, \ldots, N_{C}, q \neq j} E(j, q)\right) .
$$

The position of the Gaussian center $v_{j}$ is initialized to coincide with the center of gravity of the data in each class. The position of $v_{j}$ is then updated so that the $N_{G}$ functions cover at best the learning data in the normalized domain $[0: 1] \times$ [0:1]. For each sample $x=P^{\prime \prime}\left(I_{k}\right)$ in learning set $\mathbf{L}$, let us define $g(x)$ as the label of the Gaussian function that is the most representative of $x$. The label $g(x)$ is computed with

$$
\begin{aligned}
& g(x) \\
& =\arg \left(\max _{j=1, \ldots, N_{G}}\left\{G_{j}(x), \text { such that } G_{j} \text { is associated with class } q\right\}\right) .
\end{aligned}
$$

Let us also note $N_{L}(j)$ as the number of samples in the learning set that belongs to the domain of activation of the function $G_{j}$. The position of the center of the Gaussian function with label $g(x)$ is updated using

$$
\Delta v_{g(x)}=\frac{\alpha}{N_{L}(j)}\left(v_{g(x)}-x\right)
$$

where $\Delta v_{g(x)}$ stands for the variation of $v_{g(x)}$ and $\alpha$ is a parameter selected by the user. The process is iterated until a stable position of the Gaussians or the maximum number of iterations $K_{\max }$ is reached. The displacement of the Gaussian centers is important to correctly reposition the centers when a new Gaussien node is added to one class. In this case, the centroid of all Gaussians must be able to move to improve the covering of the learning data.

The parameter $d_{j}$ of a Gaussian function $G_{j}$ associated with class $q$ is initialized with the standard deviation of the measurement corresponding to the class $q$. The variation of $d_{j}$ is performed by a trial-error method. Indeed the dispersion $d_{j}$ is a parameter which greatly influences the shape of the different Gaussian activation areas. When the dispersion is locally modified to improve the separation between 2 Gaussians, all activation areas are affected. Therefore, it is difficult to find a systematic rule for the dispersions updating. For this reason, this updating is obtained with a trialerror method. For this purpose, the network performance is evaluated when the dispersion of each Gaussien node increases or decreases with a small increment. The variations that optimize the average performance (i.e., that minimize the average error $E_{m}$ ) is selected with

$$
\begin{aligned}
& \beta^{*} \\
& =\arg \left(\min _{\beta \in\{-1,0,1\}}\left\{E_{m}\left(d_{j}(1+\beta)\right), j=1, \ldots, N_{G}\right\}\right) .
\end{aligned}
$$

The variation $\Delta d_{j}$ of $d_{j}$ is obtained with

$$
\Delta d_{j}=\gamma \cdot\left(1+\beta^{*}\right) \cdot d_{j}
$$

where $\gamma$ is a parameter close to 0 selected by the user. The algorithm is initialized with a number of Gaussien node equal to the number of classes: $N_{G}=N_{C}$. When there exist $j=$ $1, \ldots, N_{G}$ and $q=1, \ldots, N_{G}$ such that the error $E(j, q)$ is significant (i.e. $E(j, q)>\rho$, where $\rho$ is a threshold selected by the user), a new Gaussian function associated with class $j$ is added. The aim of this Gaussian function is to decrease the error $E(j, q)$ by refining the representation of the samples of type $j$. The center of the additional function coincides with the barycenter of the samples of type $j$ clustered in class $q$. Its dispersion is initialized with the standard deviation of the same samples. Gaussian functions may be added as long as the average performance is improved or by using a test with a lower bound threshold on errors $E(j, q), j=1, \ldots, N_{G}$, $q=1, \ldots, N_{G}$.

Finally the probability $C_{q}$ that sample $x=P^{\prime \prime}\left(I_{k}\right)$ belongs to the class $q$ is computed with

$$
C_{q}(x)=\frac{G_{q}^{\prime}(x)}{\sum_{j=1}^{N_{C}} G_{j}^{\prime}(x)}, \quad q=1, \ldots, N_{C},
$$

where $G_{q}^{\prime}(x)$ is defined by

$$
G_{q}^{\prime}(x)=\max _{j=1, \ldots, N_{G}}\left\{G_{j}(x)\right.
$$

such that $G_{j}$ is associated with class $\left.q\right\}$. 


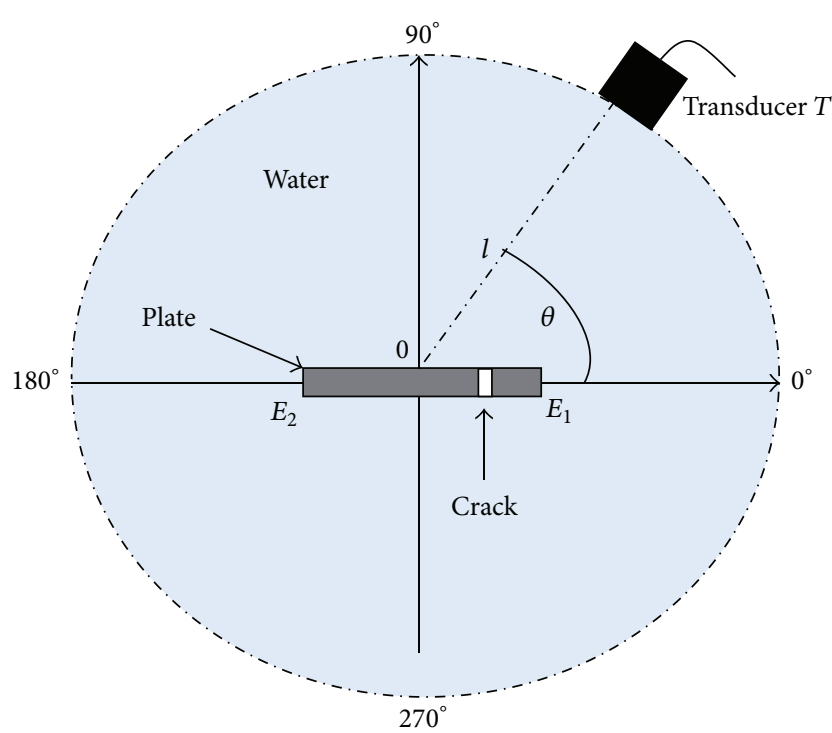

Figure 3: (Colour online) Geometrical parameter.

Finally, the class of $x=P^{\prime \prime}\left(I_{k}\right)$ is computed with respect to the maximal probability $C_{q}, q=1, \ldots, N_{C}$, with

$$
\operatorname{class}(x)=\arg \left(\max _{q=1, \ldots, N_{C}}\left\{C_{q}(x)\right\}\right) \text {. }
$$

\section{Damage Detection and Diagnosis by Classification}

3.1. Model of the Times of Flight. Let us consider a plate with two edges $E_{1}$ and $E_{2}$, a surface damage $F$, a width $L$, and a thickness $e$ to be immersed in water. It is assumed that the damage is clearly delimited and the method has been validated with cracks and holes as detailed in Section 4. The Olympus broadband transducer (V 3507) with a central frequency of $200 \mathrm{kHz}$ rotates around the plate in a plan that is orthogonal to the plate (Figure 3). It is an unfocussed immersion transducer with the lower and upper limit at $-6 \mathrm{~dB}$ that are, respectively, $0.074 \mathrm{MHz}-0.0286 \mathrm{MHz}$. Parameter $l$ is the constant distance from the transducer to the center of the plate and $\theta$ is the angle between the direction of the plate and the transducer.

In the studied frequency domain $50-400 \mathrm{kHz}$, antisymmetric $\left(A_{0}{ }^{+}\right.$and $\left.A_{0}{ }^{-}\right)$and symmetric $\left(S_{0}\right)$ Lamb waves propagate in the immersed plate in water [4]. The plate deformation is parallel to the wave propagation direction for the symmetric mode and perpendicular for the antisymmetric one. The waves reflect on the plate edges according to the Snell-Descartes laws. This last effect also appears in presence of a surface damage and produces surface acoustic Lamb waves in the plate [15]. Measured signals result from the superposition of different wave reflections in the plate. Let us note that the group and phase velocities of symmetric Lamb waves $S_{0}$ are almost constant in the explored frequency domain around $200 \mathrm{kHz}$ (Figure 4(a1)). The phase velocity of antisymmetric Lamb waves $A_{0}{ }^{-}$is smaller than the sound velocity in water $C_{w}=1470 \mathrm{~m} / \mathrm{s}$ (Figure $4(\mathrm{a} 2)$ ) whereas the phase velocity of antisymmetric Lamb waves $A_{0}{ }^{+}$is larger than $C_{w}$ [16]. For a transducer with central frequency of $200 \mathrm{kHz}$, only the waves $S_{0}$ and $A_{0}{ }^{-}$propagate and the group velocity of these waves (Figures 4 (b1) and $4(\mathrm{~b} 2)$ ) is used to determine the time of flight of the various echoes for direct and indirect trajectories (Figure 5). For simplicity, only the direct reflections $T \rightarrow E_{1} \rightarrow T$ (Figure 5(a)), $T \rightarrow$ $E_{2} \rightarrow T$ (Figure 5(b)), and $T \rightarrow F \rightarrow T$ (Figure 5(f)) are considered in the next. The time of flight for edges $E_{1}$ and $E_{2}$ is expressed by (11). It is assumed that $E_{1}$ refers to the nearest edge from the transducer. Consider

$$
\begin{aligned}
& t_{E_{1}}=\frac{2}{C_{w}} \sqrt{l^{2}+\frac{L^{2}}{4}-l L \cos (\theta)}, \\
& t_{E_{2}}=\frac{2}{C_{w}} \sqrt{l^{2}+\frac{L^{2}}{4}+l L \cos (\theta)} .
\end{aligned}
$$

Equation (12) gives the time of flight of the trajectory $T \rightarrow$ $F \rightarrow T$ generated by the damage $F$ at a distance $\delta \cdot L(0<$ $\delta<1$ ) from to $E_{1}$. Consider

$$
t_{F}=\frac{2}{C_{w}} \sqrt{l^{2}+\left(\delta-\frac{1}{2}\right)^{2} L^{2}+2 l L\left(\delta-\frac{1}{2}\right) \cos (\theta)} .
$$

The resulting model of the time of flight is given in Figure 6. This model is restricted to the incidence angle $[0: 180]$ by taking into account the plate symmetry. This model is validated in our previous work [15]. It will be used in the next to perform the useful range extraction.

3.2. Useful Range Extraction. Two neural networks similar to the ones developed in Section 2 are used to detect the echoes of the plate edges. The preprocessing stages detailed in Section 2 are first applied to these measurements. The learning set is obtained from plates with known dimensions and without any surface damage. Consequently, the measured signals contain only echoes from the plate edges. The model developed in previous section is used to extract segments that contain patterns of the plate edges and other ones. The first Gaussian neural network aims to detect the nearest edge $\left(E_{1}\right)$ with respect to the transducer position. The second one aims to detect the other edge $\left(E_{2}\right)$ that is the farthest with respect to the transducer position. Figure 7 illustrates the application of the Gaussian neural network classification used to detect the plate edge echoes of $E_{1}$ (left) and $E_{2}$ (right). Each edge is detected with a network that requires exactly $N_{G}=2$ Gaussian functions. The size of the segments is $w=50$ points. From these figures, it is clear that the first echo is easier to be detected than the second one because magnitude and energy are higher for $E_{1}$.

From the detection of edges $E_{1}$ and $E_{2}$, the useful range is extracted. Figure 8 (top) is an example of signal collected from the plate with incidence angle $\theta=20^{\circ}$. Figure 8 (bottom) details the extracted useful range. 


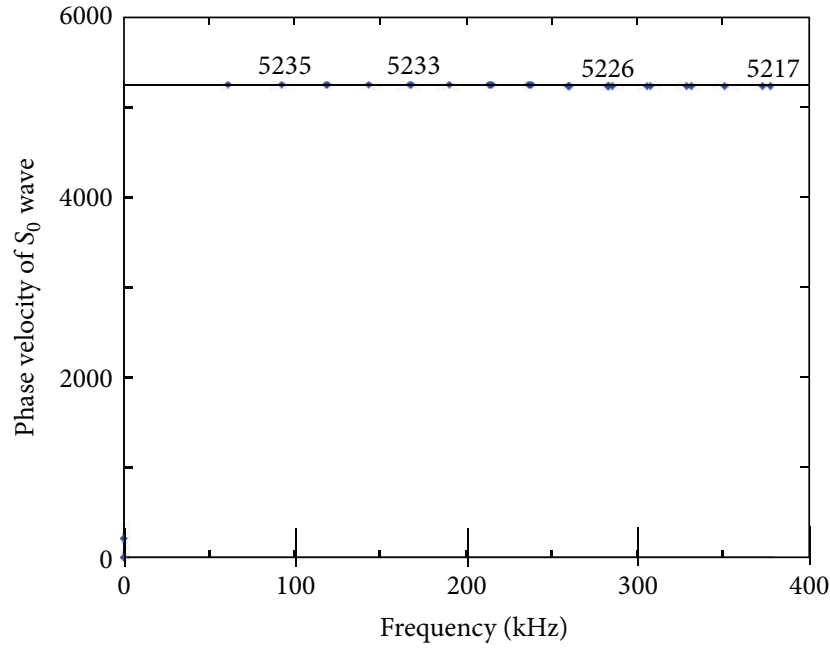

(a1)

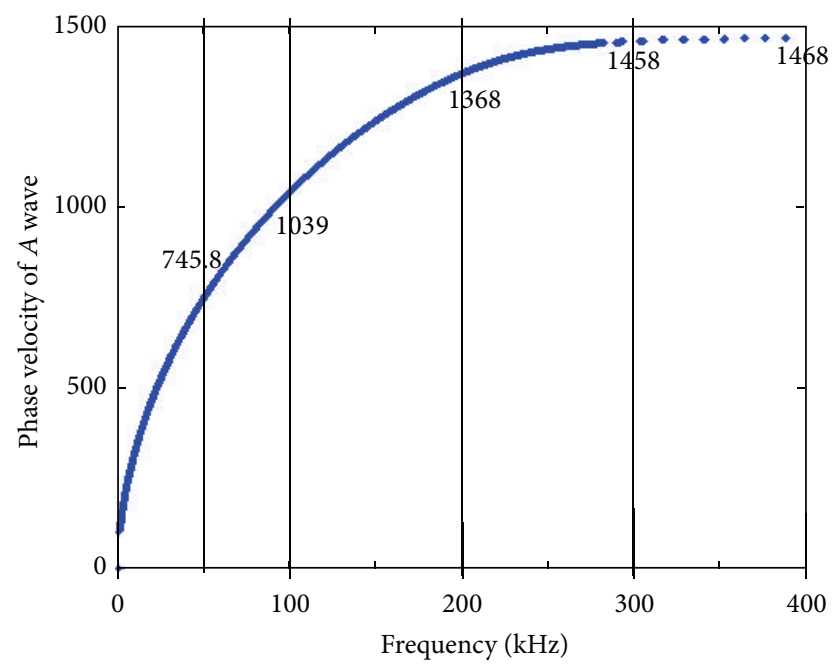

(a2)

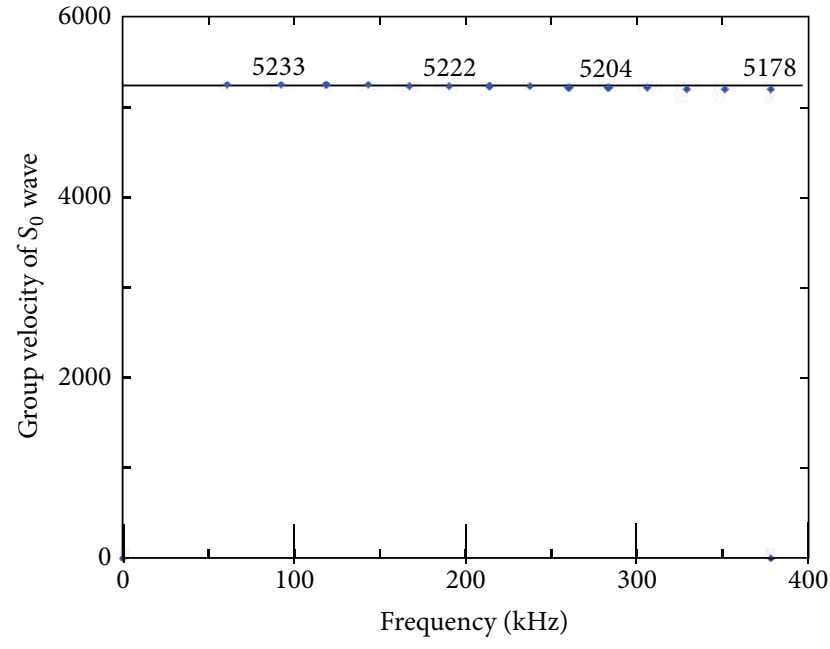

(b1)

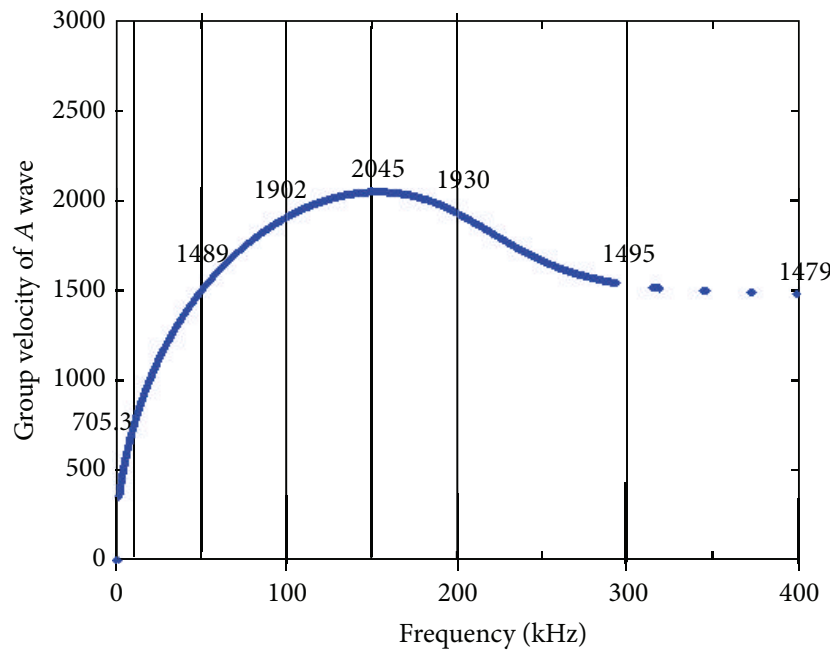

(b2)

Figure 4: (Colour online) Phase (a1) and group (b1) velocities of the symmetrical Lamb wave $S_{0}$, phase (a2), and group (b2) velocities of the antisymmetric Lamb wave $A$.

3.3. Detection and Diagnosis by Means of Classification. Two Gaussian neural network architectures (disposed in cascade) similar to the ones developed in Section 2 are used to detect and diagnose the surface damage of the structures. The efficiency of such architecture has been previously discussed and successfully compared with other usual methods $[9,10]$. For each type of damage, learning and validation sets are built using measurements obtained from healthy structures and damaged ones. First, the signal preprocessing stages are applied to these measurements. Then, a neural network with 2 classes is first used for damage detection. This network aims to separate the faulty patterns from the healthy ones. When several classes of damage exist, a second neural network is used in order to make the damage diagnosis. The full detection and diagnosis scheme is given by Figure 9. In comparison with other methods that use a complete mapping of the plate (with all incidence angles), the proposed approach is based on a small number of measurements and is more suitable for in situ analysis.

In order to improve the performance of the detection and diagnosis, several measurements of the same structure may be considered under different angles of incidence. Let us define $n_{\text {mes }}$ as the number of measurements that are used to compute the decision. In that case, the probability $C_{q}$ that the current plate belongs to the class $q$ is averaged over the $n_{\text {mes }}$ samples $x_{1}, \ldots, x_{n \text { mes }}$ that are considered. The rest of the method remains unchanged. Consider

$$
\begin{aligned}
& C_{q}\left(x_{1}, \ldots, x_{n \mathrm{mes}}\right) \\
& =\frac{1}{n_{\mathrm{mes}}}\left(\sum_{i=1, \ldots, n \mathrm{mes}}\left(\frac{G_{q}^{\prime}\left(x_{i}\right)}{\sum_{j=1}^{N_{c}} G_{j}^{\prime}\left(x_{i}\right)}\right)\right), \\
& \qquad=1, \ldots, N_{C} .
\end{aligned}
$$




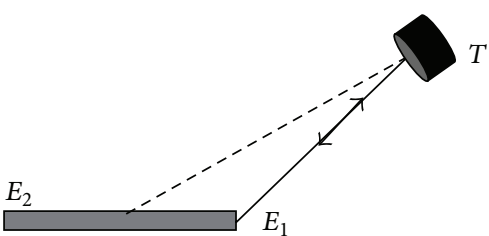

(a)

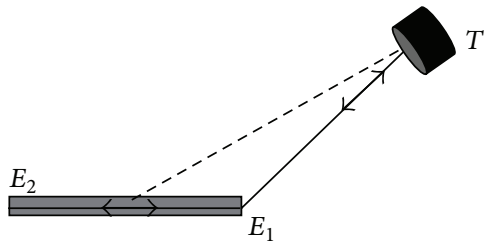

(c)

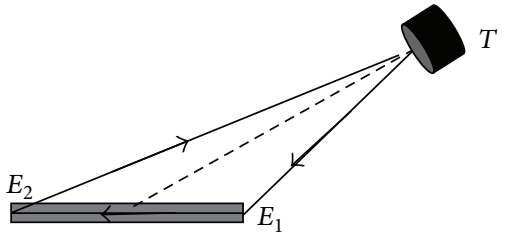

(e)

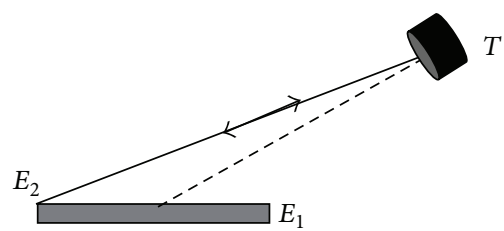

(b)

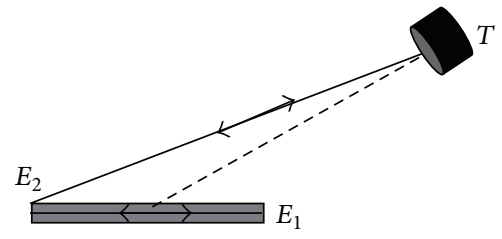

(d)

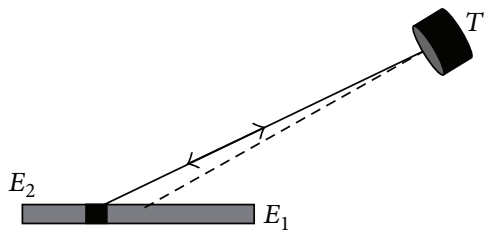

(f)

Figure 5: Different wave trajectories for an immersed plate in water.

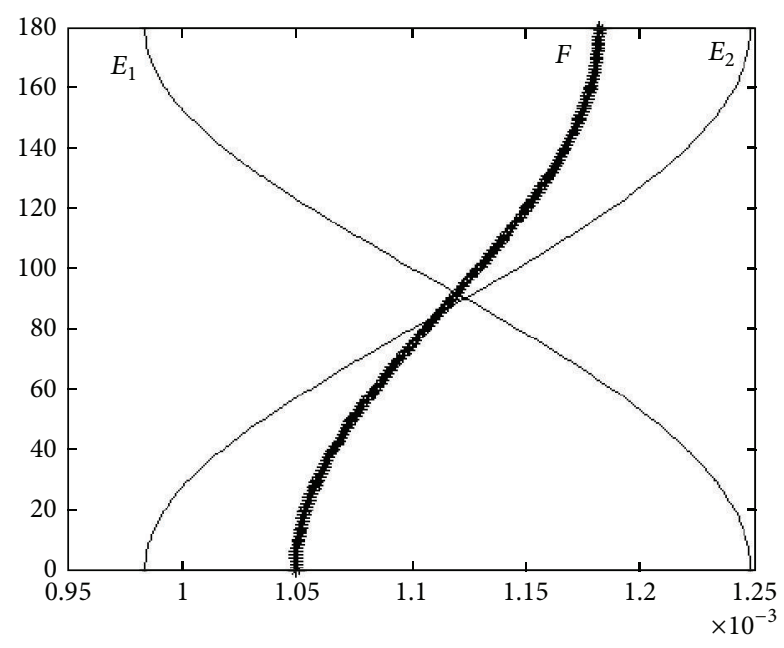

FIGURE 6: Time of flight model for an immersed plate with damage.

A confidence factor CF is also introduced with (14) in order to evaluate the likelihood of the decision provided by the Gaussian networks:

$$
\mathrm{CF}=\min \left\{\left(C_{q}-C_{q^{\prime}}\right)^{2}, q=1, \ldots, N_{C}, q^{\prime} \neq q\right\}
$$

The likelihood is computed in range $[0: 1]$ and increases up to 1 with respect to the difference between the probability of the class $q$ and the probability of the other classes $\left(q^{\prime}\right)$. This factor, used within the learning process, could be used to stop the addition of new Gaussian nodes.

\section{Application for Damage Detection and Diagnosis Using Real Data}

4.1. Experimental Setup. The experimental setup is installed in the underwater acoustic laboratory LOMC in Le Havre University. The metallic plates under investigation are suspended by two nylon threads at their upper edges in a water tank of $3 \mathrm{~m}$ diameter and $2 \mathrm{~m}$ height (Figure 10). The transducer revolves around the structures describing a circular trajectory (from $0^{\circ}$ to $360^{\circ}$ ) perpendicular to the structure main direction and the angular increment is $1^{\circ}$. For each incidence angle $\theta$, the transducer repeats several emission-acquisition operations and the collected signals are averaged in order to minimize the Signal Noise Ratio (SNR). The sampling rate is $10 \mathrm{Msamples} / \mathrm{s}$ and contains $10000 \mathrm{pts}$. The software MATLAB which stands for MATrix LABoratory is used for the signal artificial intelligence and processing.

4.2. Results Obtained for Some Simple Structures. The studied structures are currently stainless steel rectangular plates or crosses. All structures have the same physical properties. They are characterized by the longitudinal and transversal wave velocities: $C_{L}=5790 \mathrm{~m} / \mathrm{s}$ and $C_{T}=3100 \mathrm{~m} / \mathrm{s}$, respectively, and they have the same thickness $e=1.5 \mathrm{~mm}$. Three groups of structures are used (Figure 11) and the width $L$ depends on the group.

Group 1 contains 4 plates. The first plate is a reference one (REF) without any geometrical anomaly. The other plates have different classes of anomalies listed as follows: hole damage (HO), fine crack (FC), and large crack (LC). The plates in Group 1 have the same width $L=60 \mathrm{~mm}$ and the damage is located at a distance of $20 \mathrm{~mm}$ from 


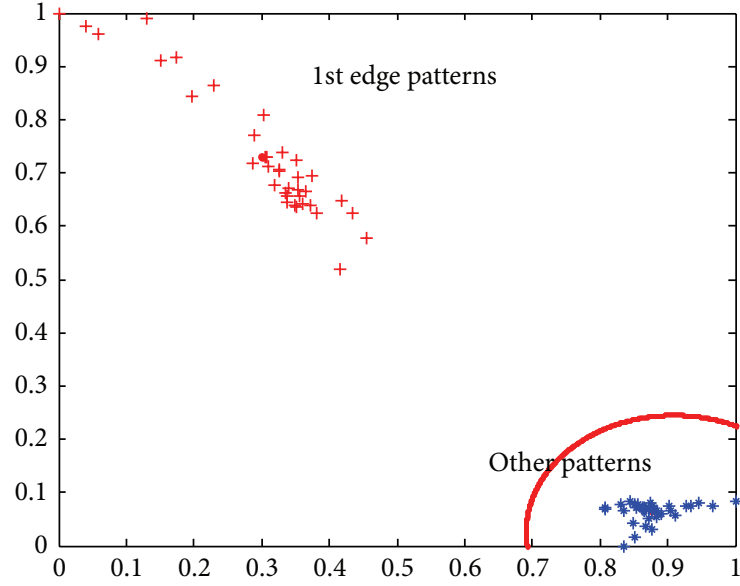

(a)

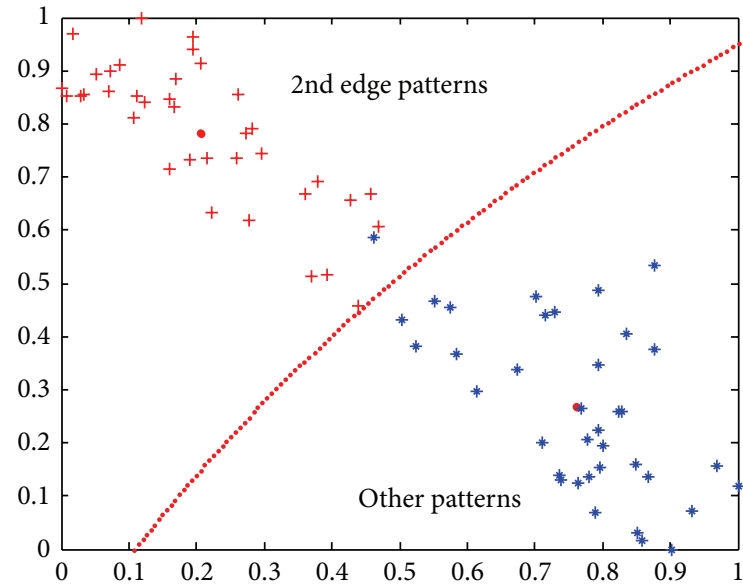

(b)

Figure 7: (Colour online) Detection of the echoes of plate edges: (a) 1st edge detection, (b) 2nd edge detection.
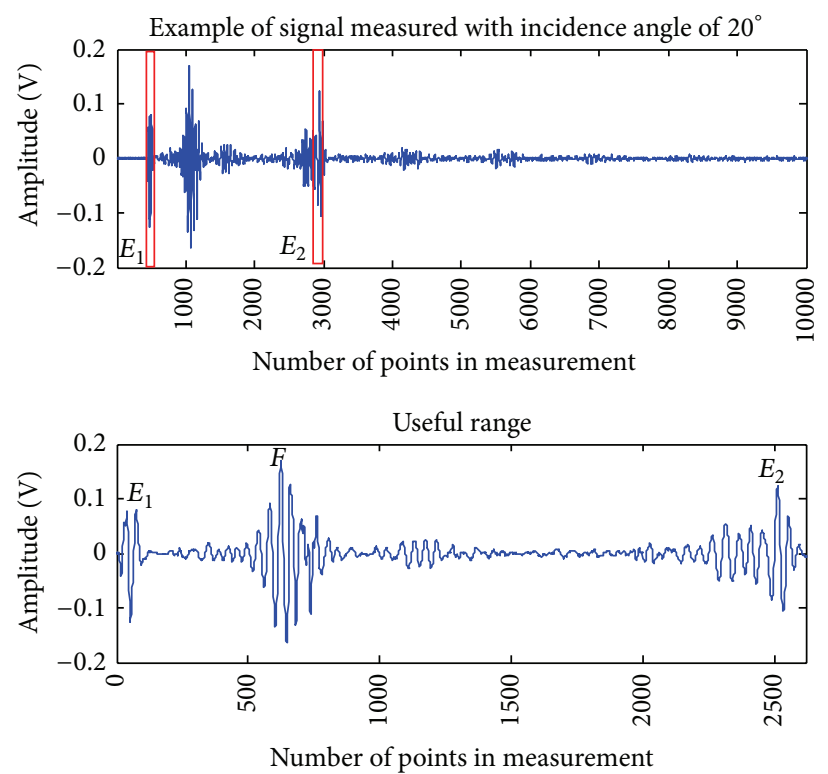

Figure 8: (Colour online) Useful range extraction.

the left plate edge. This group will be used at first to evaluate the performance of the method for diagnosis issue (i.e., identification of the class of damage).

Group 2 also contains 4 plates with width $L=194 \mathrm{~mm}$. The first plate is a reference one (REF2) without any geometrical anomaly. The other plates have the same class of anomaly: a large groove (LGR2) located at different positions in the plate (17 mm, $52 \mathrm{~mm}$, and $71 \mathrm{~mm}$, resp., from the left plate edge). This group will be used to evaluate the performance of the method for detection issue.

Group 3 contains two metallic crosses made by assembling 4 plates. The dimension of these crosses is $100 \mathrm{~mm}$. The first cross is healthy and the second one has a hole. This group will be used to illustrate that the method is suitable to detect damage in more complex geometric structures.
TABLE 1: Damage detection and diagnosis percentages for Group 1.

\begin{tabular}{lcccccc}
\hline & & \multicolumn{2}{c}{ Group 1 } & \multicolumn{3}{c}{ Diagnosis } \\
& Detection & \multicolumn{4}{c}{} \\
& Healthy & Faulty & & HO & FC & LC \\
\hline$P_{1}$ & $\mathbf{9 4}$ & 6 & $P_{2}$ & $\mathbf{9 7}$ & 3 & 0 \\
$P_{2,3,4}$ & 30 & $\mathbf{7 0}$ & $P_{3}$ & 6 & $\mathbf{8 8}$ & 6 \\
& & & $P_{4}$ & 0 & 13 & $\mathbf{8 7}$ \\
\hline
\end{tabular}

TABle 2: Damage detection percentages for Groups 2 and 3.

\begin{tabular}{lcc}
\hline & Healthy & Faulty \\
\hline & Group 2 & \\
$P_{5}$ & $\mathbf{9 9}$ & 1 \\
$P_{6,7,8}$ & 16 & $\mathbf{8 4}$ \\
\hline & Group 3 \\
$P_{9}$ & $\mathbf{7 8}$ & \\
$P_{10}$ & 19 & 22 \\
\hline
\end{tabular}

Tables 1 and 2 provide the performance of the proposed method for each group of structures. These performances have been obtained using $n_{\text {mes }}=5$ (number of measurements under different incident angles). The number of tests is 1000 for each group of structures. Note also that the segmentation has been simplified in order to focus on segments that are centered on the echoes of edges and damage. For each group, the tested structures are represented in the different rows and the classes are represented in the different columns. For example, Group 1 contains the plates $P_{1}$ to $P_{4}$ that are decided as healthy or faulty and the faulty plates are then separated into the classes of hole, fine crack, or large crack. Let us first consider the damage detection: for Group 1, the false alarm rate (i.e., the ratio of measurements from plate $P_{1}$ that are clustered as faulty) is $6 \%$ (Table 1 , left). The nondetection rate (i.e., measurement from plates $P_{2}$ to $P_{4}$ that are clustered as healthy) is $30 \%$ (Table 1 , left). This bad result is explained by the poor detection of the hole due to its size (diameter about 


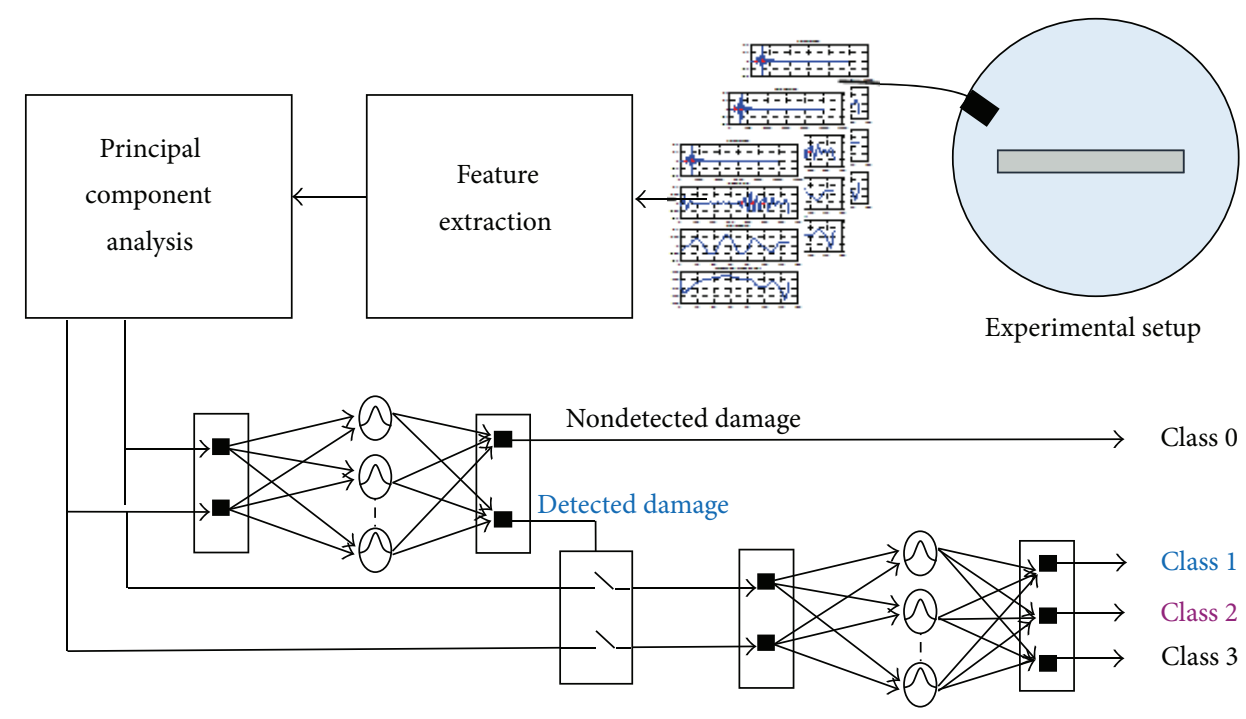

Figure 9: (Colour online) Detection and diagnosis scheme.

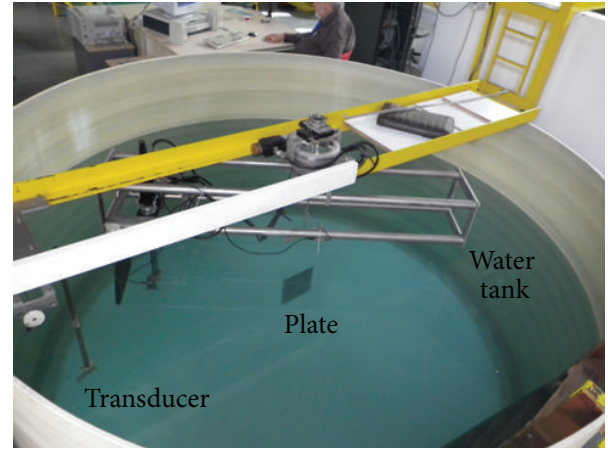

FIgURE 10: (Colour online) Experimental setup.

$1 \mathrm{~mm})$. When the hole is no longer considered, the detection rate is more than $95 \%$. The performance for damage detection of Groups 2 and 3 is given in Table 2. Let us then consider the diagnosis that is achieved for measurements issued from plates $P_{2}$ to $P_{4}$ that have been clustered as faulty (Table 1 , right). The classification rates are, respectively, $97 \%, 89 \%$, and $100 \%$ for the hole, fine crack, and large crack. Note that the proposed approach is conditioned by the parameters detailed in Table 3.

In order to evaluate the significance of the previous results, Figure 12 illustrates the performance of detection for different groups with respect to the number $n_{\text {mes }}$ of measurements. As expected, the performance increases with respect to $n_{\text {mes }}$. Let us, however, notice that the performance of Group 1 does not exceed 0.83 due to nondetection for some damage.

\section{Conclusion}

In the present study, the backscattered signals on immersed structures were analysed for damage detection and diagnosis.
TABLE 3: Used parameters.

\begin{tabular}{lcl}
\hline Parameters & Values & Definition \\
\hline$p$ & 2 & Number of principal components \\
\hline$\alpha$ & 0.6 & $\begin{array}{l}\text { Adaptation parameter for Gaussian } \\
\text { center }\end{array}$ \\
\hline$\beta$ & 0.1 & $\begin{array}{l}\text { Adaptation parameter for Gaussian } \\
\text { dispersion }\end{array}$ \\
\hline \multirow{2}{*}{50 points } & $\begin{array}{l}\text { Size of extracted segments for } \\
\text { Group 1 } \\
\text { Size of extracted segments for } \\
\text { Group 2 } \\
\text { Size of extracted segments for } \\
\text { Group 3 }\end{array}$ \\
\hline
\end{tabular}

The proposed approach is a noncontact method suitable for a large range of the incident angles. The validation of this method illustrates that the method is suitable for simple structures and can be extended to complex ones. The advantage of the proposed method lies also on its ability to analyze multivariable information under different incidence angles which makes possible in situ inspections. This method is also suitable for other materials such as composites and ceramics, which are both used in underwater applications. In contrast, note that the diagnosis of polymers cannot be achieved with this method because of its high attenuation factor to guided waves.

In our further works, we will continue the investigation of damage detection in immersed structures with artificial intelligence; we will compare results with other methods and discuss the segmentation more in detail. In particular, the influence of the window size $w$ and of the increment between two successive segments will be discussed. Improvements 

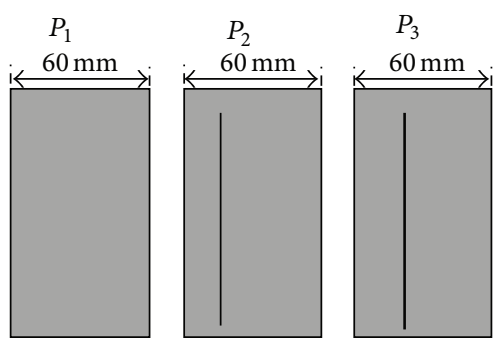

(1)
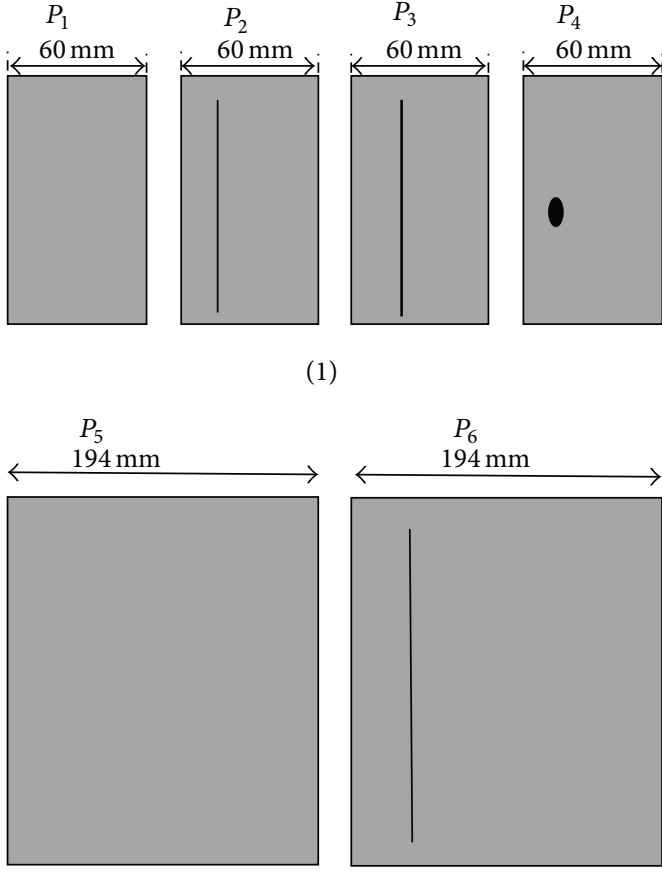

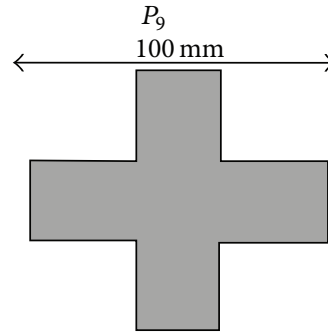

(3)
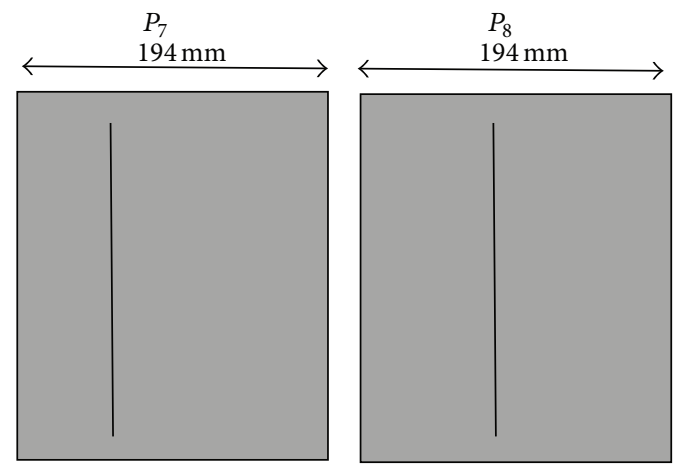

(2)

FIGURE 11: Groups of metallic structures under investigation.

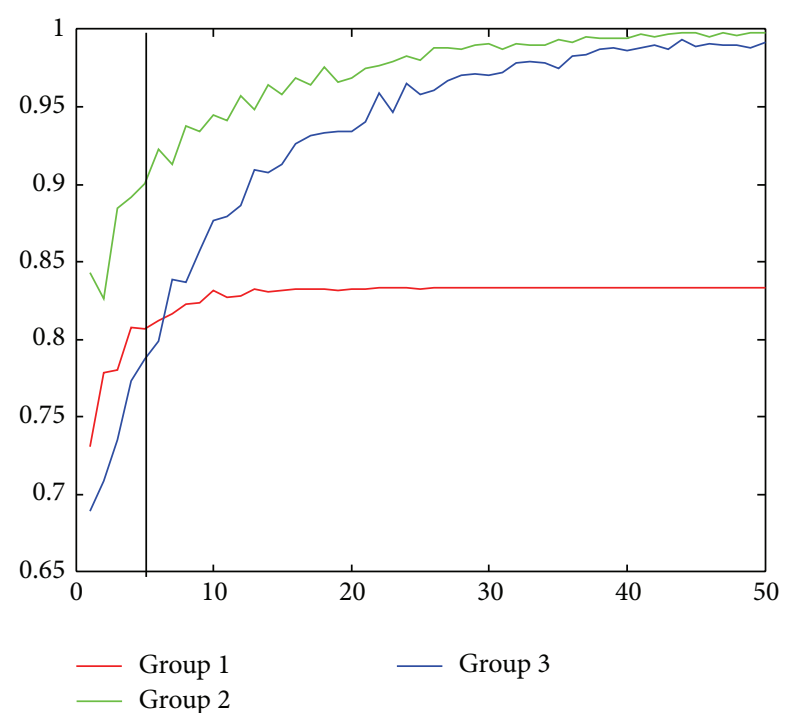

Figure 12: (Colour online) Influence of the number of measurements.

thanks to wavelet decomposition, empirical mode decomposition, and other time-frequency transformations will be also considered.

\section{Conflict of Interests}

The authors declare that there is no conflict of interests regarding the publication of this paper.

\section{Acknowledgment}

The authors are grateful to the Ministère de l'Enseignement Supérieur et de la Recherche (France) for its financial support.

\section{References}

[1] N. Mechbal, M. Vergé, G. Coffignal, and M. Ganapathi, "Application of a combined active control and fault detection scheme to an active composite flexible structure," Mechatronics, vol. 16, no. 3-4, pp. 193-208, 2006.

[2] A. Preumont, Vibration Control of Active Structures: An Introduction, Kluwer Academic Publishers, Dodrecht, The Netherlands, 2nd edition, 2002.

[3] S. P. Singh, H. S. Pruthi, and V. P. Agarwal, "Efficient modal control strategies for active control of vibrations," Journal of Sound and Vibration, vol. 262, no. 3, pp. 563-575, 2003.

[4] A. Heckman, J. Rovey, K. chandrashekhara et al., "Structural health monitoring data transmission for composite hydrokinetic turbine blades," Advanced Shipping and Ocean Engineering, vol. 2, pp. 50-59, 2013.

[5] P. Rizzo and F. L. di Scalea, "Feature extraction for defect detection in strands by guided ultrasonic waves," Structural Health Monitoring, vol. 5, no. 3, pp. 297-308, 2006.

[6] P. Rizzo, J.-G. Han, and X.-L. Ni, "Structural health monitoring of immersed structures by means of guided ultrasonic waves," Journal of Intelligent Material Systems and Structures, vol. 21, no. 14, pp. 1397-1407, 2010.

[7] S. Zhongqing and L. Ye, Identification of Damage Using Lamb Waves, Springer, Berlin, Germany, 2009. 
[8] A. Shelke, T. Kundu, U. Amjad, K. Hahn, and W. Grill, "Modeselective excitation and detection of ultrasonic guided waves for delamination detection in laminated aluminum plates," IEEE Transactions on Ultrasonics, Ferroelectrics, and Frequency Control, vol. 58, no. 3, pp. 567-577, 2011.

[9] M. Barakat, F. Druaux, D. Lefebvre, M. Khalil, and O. Mustapha, "Self adaptive growing neural network classifier for faults detection and diagnosis," Neurocomputing, vol. 74, no. 18, pp. 3865-3876, 2011.

[10] M. Barakat, D. Lefebvre, M. Khalil, F. Druaux, and O. Mustapha, "Parameter selection algorithm with self adaptive growing neural network classifier for diagnosis issues," International Journal of Machine Learning and Cybernetics, vol. 4, no. 3, pp. 217-233, 2013.

[11] M.-F. Harkat, G. Mourot, and J. Ragot, "An improved PCA scheme for sensor FDI: application to an air quality monitoring network," Journal of Process Control, vol. 16, no. 6, pp. 625-634, 2006.

[12] I. Jolliffe, Principal Component Analysis, Springer Series in Statistics, Springer, 2002

[13] H. Meng and X. Ke, "Further research on principal component analysis method of face recognition," in Proceedings of the IEEE International Conference on Mechatronics and Automation (ICMA '08), pp. 421-425, IEEE, Takamatsu, Japan, August 2008.

[14] Y. Sidibe, D. Lefebvre, F. Druaux, G. Maze, and F. Leon, "Decision tools for the diagnosis of immersed structures," Journal Européen des Systèmes Automatisés, vol. 47, no. 4-8, pp. 339-360, 2013.

[15] G. Maze, F. Léon, F. Chati et al., "Detection of a straight groove in a metal plate by acoustic scattering in water with applications to marine current turbines," NDT \& E International, vol. 65, pp. 47-53, 2014.

[16] G. Maze, N. Cité, F. Léon, F. Chati, and D. Décultot, “Acoustic scattering from a finite plate immersed in water in low frequency: influence of lamb waves A and s0," in Proceedings of the 4th International Conference and Exhibition on Underwater Acoustic Measurements: Technologies and Results (UAM '11), Kos, Greece, June 2011. 

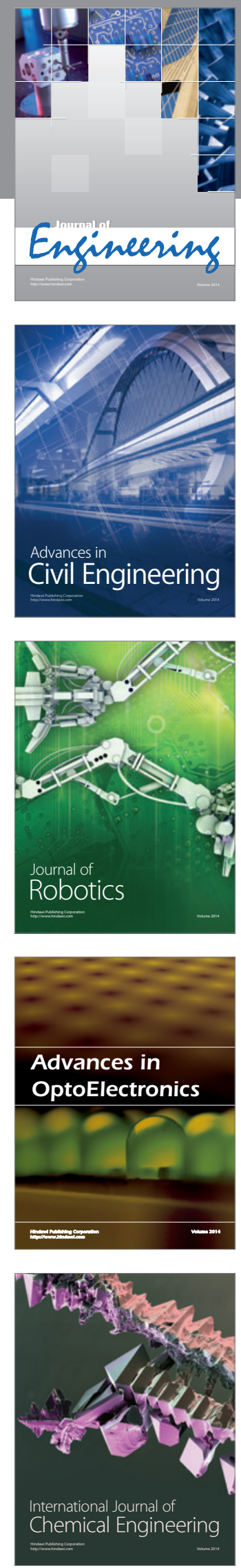

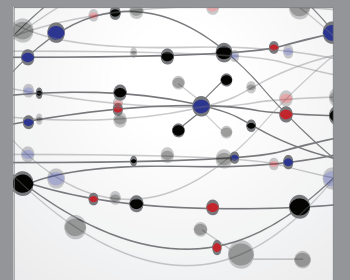

The Scientific World Journal
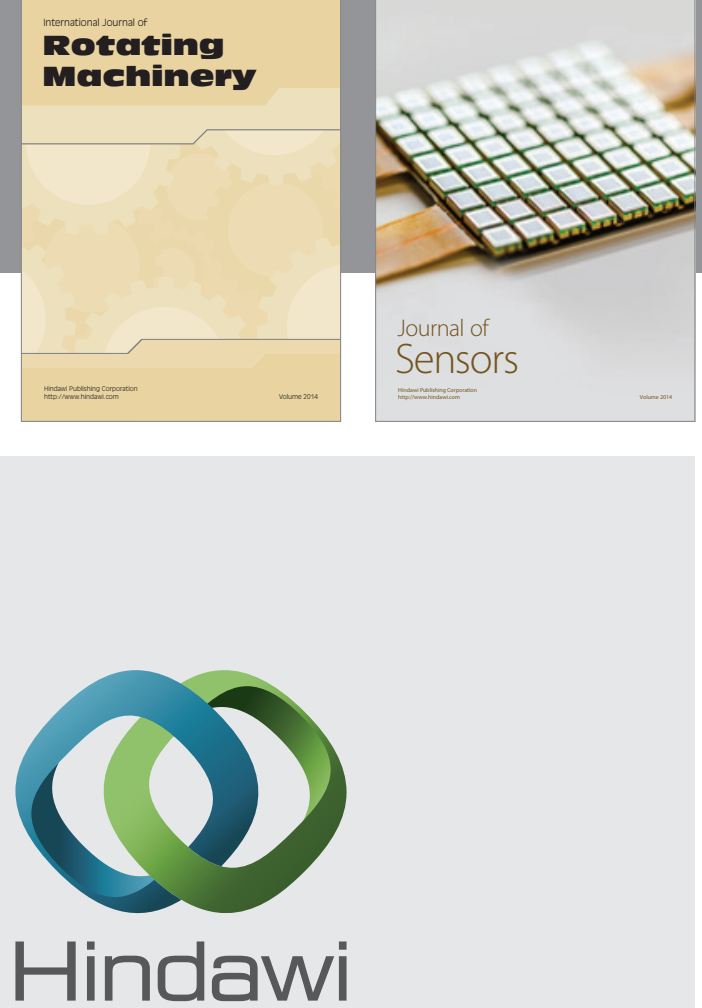

Submit your manuscripts at http://www.hindawi.com
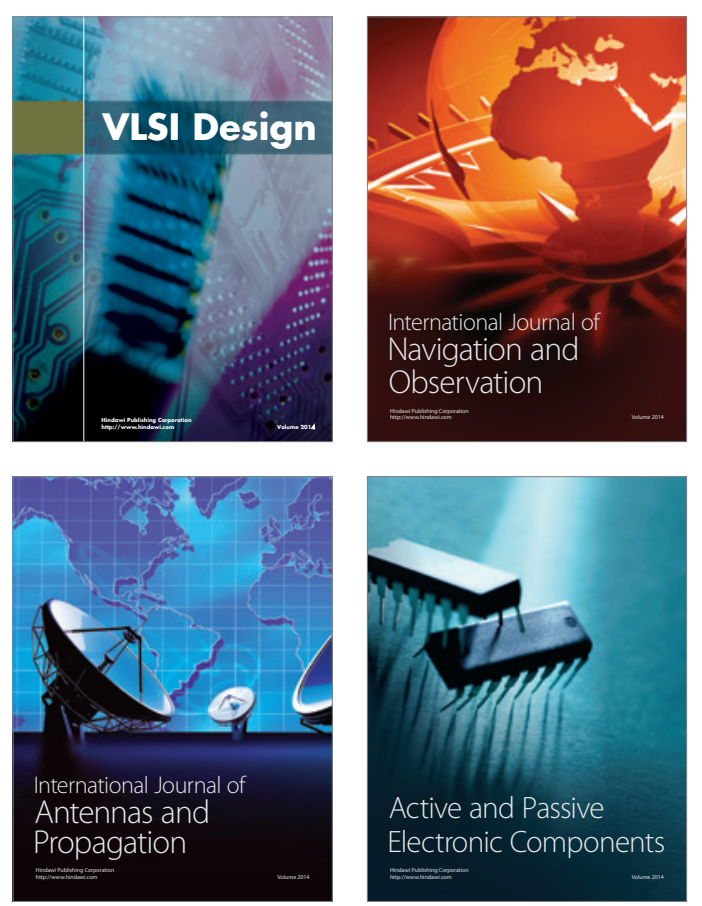
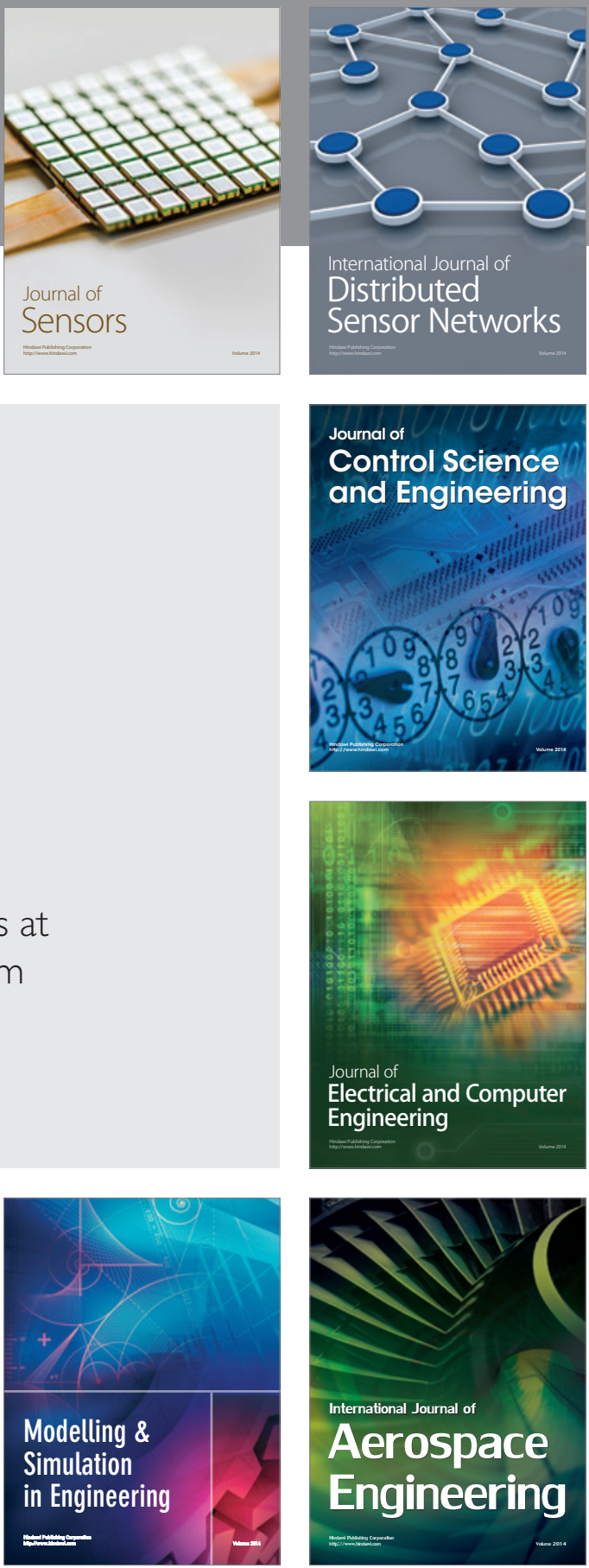

Journal of

Control Science

and Engineering
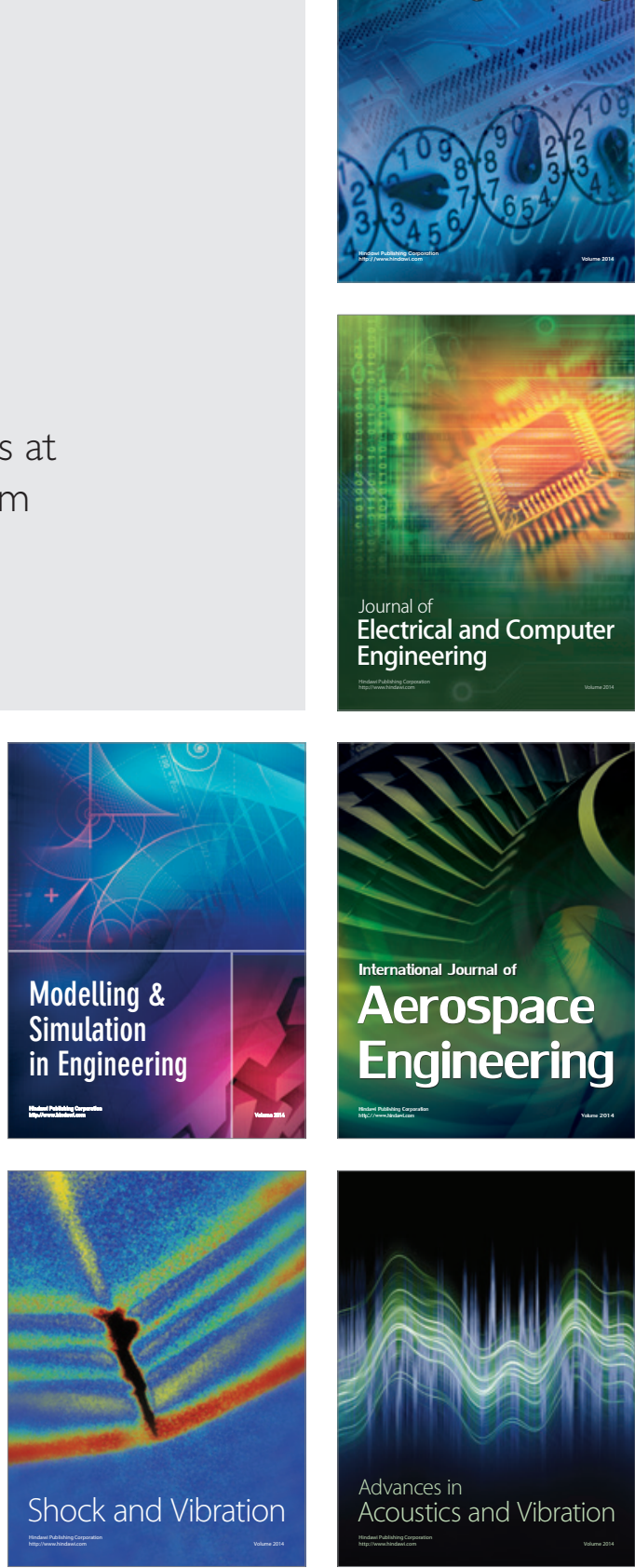